\title{
Cyclophosphamide and Etoposide as a Salvage Treatment in Metastatic Osteosarcoma Patients
}

\author{
Fatma MF Akl *, Mohamed Farouk Akl \\ Clinical Oncology and Nuclear Medicine Department, Mansoura University, Mansoura, Egypt \\ Email: ^fatmaakl@yahoo.com
}

How to cite this paper: Akl, F.M. and Akl, M.F. (2018) Cyclophosphamide and Etoposide as a Salvage Treatment in Metastatic Osteosarcoma Patients. Journal of Cancer Therapy, 9, 529-537. https://doi.org/10.4236/jct.2018.97044

Received: May 31, 2018

Accepted: July 6, 2018

Published: July 9, 2018

Copyright $\odot 2018$ by authors and Scientific Research Publishing Inc. This work is licensed under the Creative Commons Attribution International License (CC BY 4.0).

http://creativecommons.org/licenses/by/4.0/ (c) (i) Open Access

\begin{abstract}
Background and Objective: Osteosarcoma is a rare bone cancer with approximately $30 \%-35 \%$ of patients who will relapse either systemically or locally, with the lung being the commonest site of relapse. The objective of this trial was to evaluate the efficacy of cyclophosphamide and etoposide, in treatment of metastatic osteosarcoma patients progressed after one or more chemotherapy lines, with the progression free survival and treatment response as the primary endpoints, while the secondary endpoints were overall survival and treatment toxicity. Patients and Methods: Twenty seven metastatic osteosarcoma patients were enrolled into this trial and received cyclophosphamide and etoposide chemotherapy. Cyclophosphamide was given at a dose of $500 \mathrm{mg} / \mathrm{m}^{2}$ per day, I.V for 5 days and etoposide $\left(100 \mathrm{mg} / \mathrm{m}^{2}\right.$ per day I.V for 5 days). Response was assessed after 3 cycles according to Response Evaluation Criteria in Solid Tumors (RECIST) version 1.1. Chemotherapy Toxicity was graded according to the Common Terminology Criteria for Adverse Events (CTCAE). Results: The median overall survival time and progression-free survival were 12 months and 8 months, respectively. Four patients (14.8\%) achieved partial response; 14 patients (51.9\%) had stationary disease (SD); and 9 (33.3\%) expressed tumor progression. Hematologic toxicity was the main toxicity. None of the patients had G4 or life threatening toxicities. Conclusion: The combination of cyclophosphamide and etoposide represents an efficient and tolerable treatment option for patients with metastatic osteosarcoma.
\end{abstract}

\section{Keywords}

Cyclophosphamide, Etoposide, Metastatic Osteosarcoma, Salvage

Chemotherapy

\section{Introduction}

Osteosarcoma is a rare bone cancer affecting mainly adolescents and young 
adults. Most are high-grade malignancies with a high probability for lung metastases [1].

However, approximately $30 \%$ - 35\% of osteosarcoma patients will relapse either systemically or locally, with the lung being the commonest site of relapse [2].

Multi-agent chemotherapy together with surgery has improved the treatment results of patients with localized osteosarcoma [1] [2]. Treatment options for relapsed patients are limited with short survival, specifically for those with extra lung metastasis. The 4 years overall survival for patients with bone metastasis is $0 \%[3]$.

Patients with unresectable, recurrent or metastatic osteosarcoma experience poor progression-free survival. Different treatment strategies like surgery, palliative chemotherapy, radiotherapy, proton and heavy ion therapy, samarium, embolization and thermal ablation (radiofrequency and cryotherapy), were applied to control disease and prolong survival [4].

Several second-line and further line chemotherapy, like ifosfamide, etoposide, high-dose carboplatin and etoposide, topotecan, irinotecan, gemcitabine, docetaxel, imatinib mesylate, and temozolamide, have been tried, but with low response rate ranged from $3 \%$ to $29 \%$ and, more importantly, short survival time where, the median (PFS) was from 1.4 to 4 months [3] [5]. Second-line chemotherapy options are still limited and not standardized. At present, there is still no consensus on the best second-line chemotherapy [6].

Response rate of $28.5 \%$ was reported by Rodriguez-Galindo, who treated 14 patients with refractory osteosarcoma with cyclophosphamide and etoposide (cyclophosphamide $500 \mathrm{mg} / \mathrm{m}^{2} /$ day, $\mathrm{d} 1-5$ and etoposide $100 \mathrm{mg} / \mathrm{m}^{2} /$ day, $\mathrm{d} 1-5$ ) [7].

Therefore, we designed this non randomized prospective phase II trial to evaluate the activity of cyclophosphamide and etoposide combination in metastatic osteosarcoma patients, progressed after one or more chemotherapy lines, with the progression free survival and treatment response were the primary endpoints, while the secondary endpoints were overall survival and treatment toxicity.

\section{Patients and Methods}

After acceptance of the Mansoura Faculty of Medicine, institutional research board MFM IRB (code R.18.03.68), twenty-seven patients were included into this trial between July 2015 and July 2017 at the department of clinical oncology and nuclear medicine, Mansoura University Hospital, Egypt. All patients or their parents had signed the informed consent forms before enrollment.

Eligibility criteria: 1) high-grade metastatic osteosarcoma, 2) more than or equal to 18 years, 3) Eastern Cooperative Oncology Group (ECOG) performance status of $0-2,4)$ unresectable metastatic disease, 5) progressed disease after at least one line of chemotherapy, 6) availability of demographic, clinical and follow-up data, 7) normal bone marrow, renal and liver functions. 
Before study inclusion, all patients had physical examination, computerized tomography, or magnetic resonance imaging of the primary tumor and metastatic sites, and a bone scan.

Chemotherapy consisted of cyclophosphamide and etoposide, cyclophosphamide at a dose of $500 \mathrm{mg} / \mathrm{m}^{2}$ per day I.V for 5 days and etoposide $100 \mathrm{mg} / \mathrm{m}^{2}$ per day I.V for 5 days, given with granulocyte colony-stimulating factor (G-CSF) on the 7 th day for 3 days.

Response was assessed according to Response Evaluation Criteria in Solid Tumors (RECIST) version 1.1 [8]. Response assessment was done after the first 3 cycles and then every 3 - 4 cycles or as clinically indicated by computerized tomography, or magnetic resonance imaging.

Chemotherapy Toxicity data were assessed during the clinical follow up visits and graded according to the Common Terminology Criteria for Adverse Events (CTCAE) version 4 [9].

Statistical analysis: Data were entered and analyzed using SPSS software (version 21) and Medcalc software (version 15.8).

Qualitative data were expressed as count and percent.

Quantitative data were initially tested for normality using Kolmogorov-Smirnov and Shapiro-Wilk's test with data being normally distributed if $\mathrm{p}>$ 0.050 . Quantitative data were expressed as mean \pm standard deviation (SD) or median. Progression-Free Survival (PFS) and Overall Survival (OS) were analyzed by Kaplan Meier curves from the first day of treatment until disease progression (PFS) or death or last follow up (OS).

\section{Results}

This study involved 27 patients diagnosed with high grade metastatic osteosarcoma. There were 22 males (81.5\%) and 5 females (18.5\%). The median age was 20 years (range 18 - 40). ECOG performance status of grade 1 was the commonest. The femur was the commonest primary tumor site found in $22(81.5 \%) \mathrm{pa}-$ tients, followed by tibia in $3(11.1 \%)$ patients and chest wall in $2(7.4 \%)$ patients. Twenty three patients (85.2\%) had only lung metastases, while 4 (14.8\%) patients had both lung and bone metastases (Table 1$)$.

The median follow-up was 7 months ( $3-20 \mathrm{~m})$. The median number of chemotherapy cycles was $4(1-12)$.

Median OS and PFS were 12 months (95\% CI 8 - 14) and 8 months (95\% CI 4.5 - 11), respectively (Figure 1 and Figure 2).

Six months and 1 year OS were $75 \%$ and $39 \%$ respectively, while the six months and 1-year PFS were 50\% and 18\% respectively (Figure 1 and Figure 2).

No patients had complete response, 4 patients (14.8\%) of 27 expressed partial response, 14 patients (51.9\%) had stationary disease (SD), and 9 (33.3\%) of 27 had tumor progression (Table 2).

Hematologic toxicity was the main toxicity with anemia was the commonest one, none of the patients had G4 or life threatening toxicity, GI anemia was de- 
tected in $12(44.4 \%)$ patients, GII in $9(33.3 \%)$ patients and GIII in $4(14.8 \%)$ patients, GI neutropenia was encountered in 14 (51.9\%) patients, GII in 5 (18.5\%) patients and GIII in only $1(3.7 \%)$ patient, GI thrombocytopenia was found in 5 (18.5\%) patients and GII in only 2 (7.4\%) patients (Table 3 ).

Alopecia was the commonest non hematologic toxicity where 4 (14.8\%) patients had GI and 23 (85.2\%) patients were of GII. Six (22.2\%) patients experienced GI nausea and only 2 (7.4\%) patients had GI cystitis (Table 3 ).

Table 1. Patients characteristics.

\begin{tabular}{cc}
\hline Characteristics & No. (\%) \\
\hline Age (years) & 20 \\
Median & $(18-40)$ \\
Range & $22(81.5 \%)$ \\
Sex & $5(18.5 \%)$ \\
Male & \\
Female & $8(29.6 \%)$ \\
ECOG performance status & $13(48.2 \%)$ \\
0 & $6(22.2 \%)$ \\
1 & \\
2 & $22(81.5 \%)$ \\
1ry tumor site & $3(11.1 \%)$ \\
Femur & $2(7.4 \%)$ \\
Tibia & \\
Chest wall & $23(85.2 \%)$ \\
Metastatic site & $4(14.8)$ \\
Lung & 4 \\
Lung and Bone & $(1-12)$ \\
N of chemotherapy cycles & \\
Median & \\
Range &
\end{tabular}

Table 2. Tumor response.

\begin{tabular}{ccc}
\hline Response & No. & $\%$ \\
\hline Partial response & 4 & 14.8 \\
Stable disease & 14 & 51.9 \\
Progressive disease & 9 & 33.3 \\
\hline
\end{tabular}

Table 3. Treatment-related toxicity.

\begin{tabular}{ccccccccc}
\hline \multirow{2}{*}{ Toxicities } & \multicolumn{2}{c}{ Grade I } & \multicolumn{2}{c}{ Grade II } & \multicolumn{2}{c}{ Grade III } & \multicolumn{2}{c}{ Grade IV } \\
\cline { 2 - 9 } & No. & $\%$ & No. & $\%$ & No. & $\%$ & No. & $\%$ \\
\hline Haematological & & & & & & & & \\
Anaemia & 12 & 44.4 & 9 & 33.3 & 4 & 14.8 & 0 & 0 \\
Neutropenia & 14 & 51.9 & 5 & 18.5 & 1 & 3.7 & 0 & 0 \\
Thrombocytopenia & 5 & 18.5 & 2 & 7.4 & 0 & 0 & 0 & 0 \\
Non-hematological & & & & & & & & \\
Nausea & 6 & 22.2 & 0 & 0 & 0 & 0 & 0 & 0 \\
Alopecia & 4 & 14.8 & 23 & 85.2 & 0 & 0 & 0 & 0 \\
Cystitis & 2 & 7.4 & 0 & 0 & 0 & 0 & 0 & 0 \\
\hline
\end{tabular}




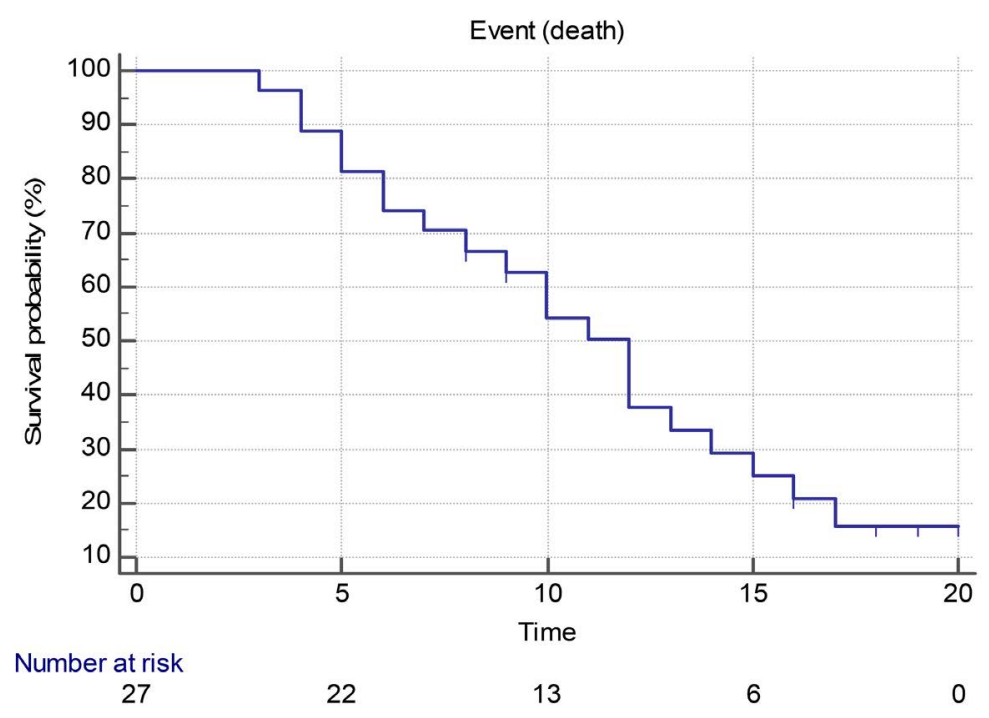

Figure 1. Overall survival curve.

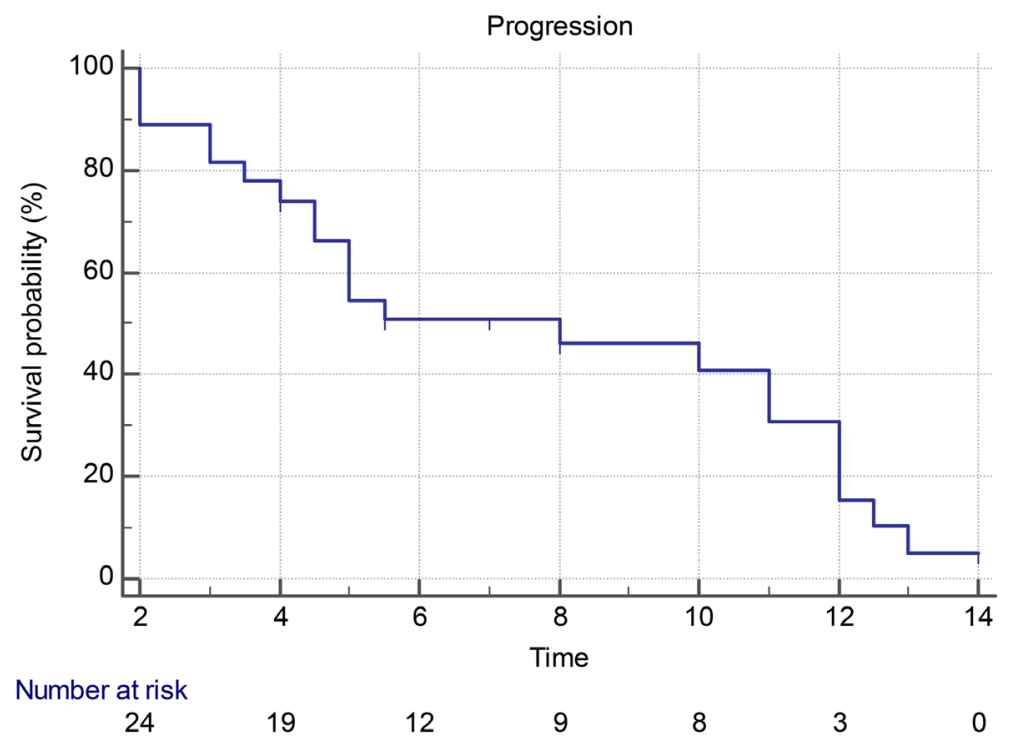

Figure 2. Progression free survival curve.

The effect of different factors (age, sex, PS, primary tumor site, metastatic sites and number of chemotherapy cycles) on OS and PFS was studied and revealed that the only significant factor which affected survival was performance status (OS $\mathrm{p}=0.04$, PFS $\mathrm{p}=0.006)$.

\section{Discussion}

Despite multimodality treatments, about one third of localized osteosarcoma patients, as well as nearly $75 \%$ of metastatic patients at diagnosis relapse [10].

No accepted standard second-line chemotherapy for recurrent osteosarcoma is established. Choice of chemotherapy depends on the length of previous progression free survival, and mostly, it includes ifosfamide, etoposide, carboplatin 
and other active agents [6].

In this phase II non randomized study, we evaluated the efficacy of cyclophosphamide and etoposide in metastatic osteosarcoma patients progressed after one or more lines of chemotherapy.

Our study demonstrated the median OS and PFS of 12 and 8 months, respectively. Six months and 1 year OS were $75 \%$ and $39 \%$ respectively, while the six months and 1-year PFS were 50\% and 18\% respectively. Regarding response, 4 patients (14.8\%) of 27 expressed partial response, 14 (51.9\%) had stationary disease (SD), and $9(33.3 \%)$ of 27 had tumor progression. Hematologic toxicity was the main toxicity with anemia was the commonest one, none of the patients had G4 or life threatening toxicity, GI anemia was detected in $12(44.4 \%)$ patients, GII in $9(33.3 \%)$ patients and GIII in 4 (14.8\%) patients, GI neutropenia was encountered in $14(51.9 \%)$ patients, GII in $5(18.5 \%)$ patients and GIII in only 1 (3.7\%) patient, GI thrombocytopenia was found in 5 (18.5\%) patients and GII in only $2(7.4 \%)$ patients. Alopecia was the commonest non hematologic toxicity where 4 (14.8\%) patients had GI and 23 (85.2\%) patients were of GII. Six (22.2\%) patients experienced GI nausea and only $2(7.4 \%)$ patients had GI cystitis.

Our reported results are comparable with those of a phase II trial carried out by Massimo et al., who treated 26 metastatic osteosarcoma patients (lung metastasis) with cyclophosphamide at dose of $4 \mathrm{~g} / \mathrm{m}^{2}$ on Day 1 and etoposide at 200 $\mathrm{mg} / \mathrm{m}^{2}$ on Days 2, 3, and 4. Four months progression-free survivals were $42 \%$. Fever was the only grade 4 non-hematological toxicity (5\%), bronchospasm (4\%) and oral mucositis (18\%). Nineteen percent of patients expressed response, 9 had stationary disease (35\%), and 12 showed progressive disease (46\%). The one year OS was $50 \%$ [3].

In a study by Mantadakis, it involved osteosarcoma patients, treated by combination of cyclophosphamide and etoposide (cyclophosphamide at a dose of $500 \mathrm{mg} / \mathrm{m}^{2}$ daily, $\mathrm{d} 1-5$ and etoposide $\left.100 \mathrm{mg} / \mathrm{m}^{2} / \mathrm{day}, \mathrm{d} 1-5\right)$. The median overall survival time was 11 months, approximately similar to this study [11].

In contrast to most trials, Saleh et al. registered extremely higher response rate of $88 \%$ in osteosarcoma patients received cyclophosphamide and etoposide as a 1st line (cyclophosphamide of $300 \mathrm{mg} / \mathrm{m}^{2}$ twice daily for 6 doses, and etoposide $200 \mathrm{mg} / \mathrm{m}^{2} /$ day, d1-3 [12].

Also, Rodriguez-Galindo treated 14 patients with refractory osteosarcoma with cyclophosphamide and etoposide (cyclophosphamide $500 \mathrm{mg} / \mathrm{m}^{2} /$ day, d1-5 and etoposide $\left.100 \mathrm{mg} / \mathrm{m}^{2} / \mathrm{day}, \mathrm{d} 1-5\right)$ the responserate was $28.5 \%$, higher than that reported in this study (19\%) [7].

In a study that assessed the efficacy of gemcitabine, docetaxel in 51 relapsed high-grade osteosarcoma patients, they received gemcitabine at a dose of 900 $\mathrm{mg} / \mathrm{m}^{2} \mathrm{~d}$ land 8 ; docetaxel $75 \mathrm{mg} / \mathrm{m}^{2} \mathrm{~d} 8$, every 3 weeks; 4 month PFS was $46 \%$; 46 patients had measurable disease by RECIST criteria assessment: 6 patients showed partial response; 20 had stationary response and 20 developed progression. One year OS was 30\%. Their results are nearly similar to the present study. 
Grade 4 hematological toxicity was detected in 13 (25\%) patients, with $11(21 \%)$ experienced G4 neutropenia and 2 had G4 thrombocytopenia. Non-hematological toxicity was experienced in $8(16 \%)$ cases, with $3(6 \%)$ developed hyper senstivity reactions. G1 Diarrhea was recorded in 2 patients, lung fibrosis, Steven Johnson syndrome and capillary leak syndrome in one patient each, representing higher incidence and grades of toxicity in comparison to the present study [13].

These results were also in agreement with those reported in a phase II non-randomized trial that evaluated sorafenib and everolimus in progressed osteosarcoma patients. Median PFS was 5 months and 6 months PFS was 45\%. Median OS time was 11 months with 14 out of 38 (37\%) patients were alive at 1 year and the 2-year OS was 5\%. The commonest G3 - 4 adverse events were lymphopenia and hypophosphataemia each in $6(16 \%)$ patients, hand and foot syndrome in $5(13 \%)$, thrombocytopenia in $4(11 \%)$, and oral mucositis, diarrhoea, and anaemia each in $2(5 \%)$. One patient (3\%) had G3 pneumothorax that required intervention. This was reported as a serious toxicity related to the used drugs, denoting serious and more advanced grades of toxicity in comparison to the current study [14].

Song et al. retrospectively reviewed the data of 28 patients ( 20 male, 8 female) diagnosed with recurrent or refractory osteosarcoma, treated with gemcitabine (675 or $900 \mathrm{mg} / \mathrm{m}^{2}$ on days 1 and 8 ) and docetaxel $\left(100 \mathrm{mg} / \mathrm{m}^{2}\right.$ on day 8 ) at Korea Cancer Center Hospital. Eleven patients received adjuvant gemcitabine, docetaxel after surgery. Seventeen patients received gemcitabine, docetaxel as palliative treatment. They detected $24 \%$ response rate and median OS of 9 months, slightly lower than that of the current study (12 months). The 1 year OS for the adjuvant group in contrast to the palliative group was $(72.7 \% \pm 13.4 \%$ vs. $35.3 \%$ $\pm 11.6 \%, \mathrm{p}=0.006)$ [5].

Compared to other expensive chemotherapeutic agents used in this setting, cyclophosphamide, etoposide combination represents comparable results with lesser toxicity profile, which is an important issue in with low income countries.

\section{Conclusion}

In conclusion, the combination of cyclophosphamide and etoposide is an efficient and tolerable protocol in addition to its low cost, representing a suitable treatment option for patients with metastatic osteosarcoma, but further large efforts are needed to improve chemotherapeutic and surgical treatments that can be offered to these patients.

\section{Limitations of the Study}

Non randomized, small cohort.

\section{Conflict of Interests}

No conflict of interest. 


\section{References}

[1] Bielack, S.S., Hecker-Nolting, S., Blattmann, C. and Kager, L. (2016) Advances in the Management of Osteosarcoma. F1000Research. https://doi.org/10.12688/f1000research.9465.1

[2] Bacci, G., Briccoli, A., Longhi, A., Ferrari, S., Mercuri, M., Faggioli, F., Versari, M. and Picci, P. (2005) Treatment and Outcome of Recurrent Osteosarcoma: Experience at Rizzoli in 235 Patients Initially Treated with Neoadjuvant Chemotherapy. Acta Oncologica, 44, 748-755. https://doi.org/10.1080/02841860500327503

[3] Massimo, B., Giovanni, G., Stefano, F., Eleonora, B., Adalberto, B.P., Sandra, A., Francesco, S., Massimo, A. and Franca, F. (2009) Phase 2 Trial of Two Courses of Cyclophosphamide and Etoposide for Relapsed High-Risk Osteosarcoma Patients. Cancer, 115, 2980-2987. https://doi.org/10.1002/cncr.24368

[4] Errani, C., Longhi, A., Rossi, G., Rimondi, E., Biazzo, A., Toscano, A., Alì, N., Ruggieri, P., Alberghini, M., Picci, P., Bacci, G. and Mercuri, M. (2011) Palliative Therapy for Osteosarcoma. Expert Review of Anticancer Therapy, 11, 217-227. https://doi.org/10.1586/era.10.172

[5] Song, B.S., Seo, J., Kim, D.H., Lim, J.S., Yoo, J.Y. and Lee, J.A. (2014) Gemcitabine and Docetaxel for the Treatment of Children and Adolescents with Recurrent or Refractory Osteosarcoma: Korea Cancer Center Hospital Experience. Pediatric Blood and Cancer, 61, 1376-1381. https://doi.org/10.1002/pbc.25035

[6] ESMO/European Sarcoma Network Working Group (2014) Bone Sarcomas: ESMO Clinical Practice Guidelines for Diagnosis, Treatment and Follow-Up. Annals of Oncology, 25, iii113-iii123. https://doi.org/10.1093/annonc/mdu256

[7] Rodriguez-Galindo, C., Daw, N.C., Kaste, S.C., Meyer, W.H., Dome, J.S., Pappo, A.S., Rao, B.N. and Pratt, C.B. (2002) Treatment of Refractory Osteosarcoma with Fractionated Cyclophosphamide and Etoposide. Journal of Pediatric Hematology/ Oncology, 24, 250-255. https://doi.org/10.1097/00043426-200205000-00006

[8] Eisenhauer, E.A., Therasse, P., Bogaerts, J., Schwartz, L.H., Sargent, D., Ford, R., Dancey, J., Arbuck, S., Gwyther, S., Mooney, M., Rubinstein, L., Shankar, L., Dodd, L., Kaplan, R., Lacombe, D. and Verweij, J. (2009) New Response Evaluation Criteria in Solid Tumours: Revised RECIST Guideline (Version 1.1). European Journal of Cancer, 45, 228-247. https://doi.org/10.1016/j.ejca.2008.10.026

[9] US Department of Health and Human Services (2009) Common Terminology Criteria for Adverse Events (CTCAE) Version 4.0.

[10] Briccoli, A., Rocca, M., Salone, M., Guzzardella, G.A., Balladelli, A. and Bacci, G. (2010) High Grade Osteosarcoma of the Extremities Metastatic to the Lung: Long-Term Results in 323 Patients Treated Combining Surgery and Chemotherapy, 1985-2005. Surgical Oncology, 19, 193-199. https://doi.org/10.1016/j.suronc.2009.05.002

[11] Mantadakis, E., Herrera, L., Leavey, P.J., Bash, R.O., Winick, N.J. and Kamen, B.A. (2000) Fractionated Cyclophosphamide and Etoposide for Children with Advanced or Refractory Solid Tumors: A Phase II Window Study. Journal of Clinical Oncology, 18, 2576-2581. https://doi.org/10.1200/JCO.2000.18.13.2576

[12] Saleh, R.A., Graham-Pole, J., Cassano, W., Abbot, F., Griend, V.R., Dicksoin, N., Metha, P., Heare, M., Kedar, A., Heahe, T. and Gross, S. (1990) Response of Osteogenic Sarcoma to the Combination of Etoposide and Cyclophosphamide as Neoadjuvant Chemotherapy. Cancer, 65, 861-865.

https://doi.org/10.1002/1097-0142(19900215)65:4<861::AID-CNCR2820650405>3.0 .CO;2-H 
[13] Palmerini, E., Jones, R.L., Marchesi, E., Paioli, A., Cesari, M., Longhi, A., Meazza, C., Coccoli, L., Fagioli, F., Asaftei, S., Grignani, G., Tamburini, A., Pollack, S.M., Picci, P. and Ferrari, S. (2016) Gemcitabine and Docetaxel in Relapsed and Unresectable High-Grade Osteosarcoma and Spindle Cell Sarcoma of Bone. BMC Cancer, 16, 280. https://doi.org/10.1186/s12885-016-2312-3

[14] Grignani, G., Palmerini, E., Ferraresi, V., D’Ambrosio, L., Bertulli, R., Asaftei, S.D., Tamburini, A., Pignochino, Y., Sangiolo, D., Marchesi, E., Capozzi, F., Biagini, R., Gambarotti, M., Fagioli, F., Casali, P.G., Picci, P., Ferrari, S. and Aglietta, M. (2015) Sorafenib and Everolimus for Patients with Unresectable High-Grade Osteosarcoma Progressing after Standard Treatment: A Non-Randomised Phase 2 Clinical Trial. The Lancet Oncology, 16, 98-107. https://doi.org/10.1016/S1470-2045(14)71136-2 